\title{
Nielsen type numbers and homotopy minimal periods for maps on solvmanifolds with $\mathrm{Sol}_{1}^{4}$-geometry
}

Jang Hyun Jo and Jong Bum Lee*

${ }^{*}$ Correspondence: jlee@sogang.ac.kr Department of Mathematics, Sogang University, Seoul, 04107, Korea

\begin{abstract}
For all maps $f$ on the special solvmanifolds with Sol ${ }_{1}^{4}$-geometry, we give explicit formulas for a complete computation of the Nielsen type numbers $\mathrm{NP}_{n}(f)$ and $\mathrm{N} \Phi_{n}(f)$. We also give a complete description of the sets of homotopy minimal periods of all such maps.
\end{abstract}

MSC: Primary 55M20; secondary 57S30

Keywords: homotopy minimal periods; Nielsen numbers; Nielsen type numbers; Sol ${ }_{1}^{4}$; solvmanifolds

\section{Introduction}

For a self-map $f: X \rightarrow X$, the Lefschetz number $L(f)$ and the Nielsen number $N(f)$ give information concerning the fixed points of $f$. It is known that the Nielsen number gives more precise information about the existence of fixed points than the Lefschetz number, but its computation is in general very difficult. For the periodic points, two Nielsen type numbers $\mathrm{NP}_{n}(f)$ and $\mathrm{N} \Phi_{n}(f)$ were introduced by Jiang [1], which are lower bounds for the number of periodic points of least period exactly $n$ and the set of periodic points of period $n$, respectively.

It is obvious that these Nielsen numbers are much more powerful than the Lefschetz numbers in describing the periodic point sets of self-maps. Using fiber techniques on nilmanifolds and some solvmanifolds, Heath and Keppelmann [2] (see also [3]) succeeded in showing that the Nielsen numbers and the two Nielsen type numbers are related to each other under certain conditions. However, the computation of the Nielsen type numbers even on low dimensional infra-homogeneous spaces is a hard problem. See [4] for the Klein bottle and [5] for a three-dimensional flat Riemannian manifold.

One of the natural problems in dynamical systems is the study of the existence of periodic points of least period exactly $n$. Homotopically, a new concept, namely homotopy minimal periods,

$$
\operatorname{HPer}(f)=\bigcap_{g \simeq f}\left\{n \in \mathbb{N} \mid P_{n}(g) \neq \emptyset\right\},
$$

(c) 2015 Jo and Lee. This article is distributed under the terms of the Creative Commons Attribution 4.0 International License (http://creativecommons.org/licenses/by/4.0/), which permits unrestricted use, distribution, and reproduction in any medium, provided you give appropriate credit to the original author(s) and the source, provide a link to the Creative Commons license, and indicate if changes were made. 
where $P_{n}(g)=\operatorname{Fix}\left(g^{n}\right)-\bigcup_{k<n} \operatorname{Fix}\left(g^{k}\right)$, was introduced by Alsedà et al. [6]. Since the homotopy minimal period is preserved under a small perturbation of a self-map $f$ on a manifold $X$, we can say that the set $\operatorname{HPer}(f)$ of homotopy minimal periods of $f \operatorname{describes}$ the rigid part of dynamics of $f$. A complete description of the set of homotopy minimal periods of all self-maps was obtained on the nilmanifolds with $\mathrm{Nil}^{3}$-geometry $[7,8]$ and on the special solvmanifolds with $\mathrm{Sol}^{3}$-geometry $[9,10]$.

There are four-dimensional geometries which were classified by Filipkiewicz [11], see also [12]. One of their model spaces is a simply connected four-dimensional unimodular solvable Lie group $\mathrm{Sol}_{1}^{4}$. This group contains $\mathrm{Nil}^{3}$ as a nil-radical and the quotient by its center is $\mathrm{Sol}^{3}$. Recall that $\mathrm{Nil}^{3}$ and $\mathrm{Sol}^{3}$ are model spaces for three-dimensional geometries.

In this paper, we are concerned with the special solvmanifolds with $\mathrm{Sol}_{1}^{4}$-geometry, i.e., the closed manifolds $\Gamma \backslash \mathrm{Sol}_{1}^{4}$ which are quotient spaces of $\mathrm{Sol}_{1}^{4}$ by its lattices $\Gamma$. For all continuous maps $f$ on any special solvmanifolds with $\mathrm{Sol}_{1}^{4}$-geometry, we will give a complete description of the Nielsen type numbers $\mathrm{NP}_{n}(f)$ and $\mathrm{N} \Phi_{n}(f)$, and the homotopy minimal periods $\operatorname{HPer}(f)$.

\section{The Lie group Sol ${ }_{1}^{4}$ and its Lie algebra}

Consider the connected and simply connected four-dimensional matrix Lie group

$$
\operatorname{Sol}_{1}^{4}=\left\{\left[\begin{array}{ccc}
1 & y & x \\
0 & e^{\theta} & z \\
0 & 0 & 1
\end{array}\right]: x, y, z, \theta \in \mathbb{R}\right\} .
$$

This Lie group is one of the four-dimensional geometries which were classified by Filipkiewicz [11], see also [12]. The Lie algebra of $\mathrm{Sol}_{1}^{4}$ is

$$
\mathfrak{s o r}_{1}^{4}=\left\{\left[\begin{array}{ccc}
0 & b & a \\
0 & \theta & c \\
0 & 0 & 0
\end{array}\right]: a, b, c, \theta \in \mathbb{R}\right\} .
$$

Denote

$$
\begin{array}{lll}
\mathbf{e}_{1}=\left[\begin{array}{lll}
0 & 0 & 1 \\
0 & 0 & 0 \\
0 & 0 & 0
\end{array}\right], & \mathbf{e}_{2}=\left[\begin{array}{lll}
0 & 1 & 0 \\
0 & 0 & 0 \\
0 & 0 & 0
\end{array}\right], \\
\mathbf{e}_{3}=\left[\begin{array}{lll}
0 & 0 & 0 \\
0 & 0 & 1 \\
0 & 0 & 0
\end{array}\right], & \mathbf{e}_{4}=\left[\begin{array}{lll}
0 & 0 & 0 \\
0 & 1 & 0 \\
0 & 0 & 0
\end{array}\right] .
\end{array}
$$

Its nontrivial brackets are

$$
\left[\mathbf{e}_{2}, \mathbf{e}_{3}\right]=\mathbf{e}_{1}, \quad\left[\mathbf{e}_{2}, \mathbf{e}_{4}\right]=\mathbf{e}_{2}, \quad\left[\mathbf{e}_{3}, \mathbf{e}_{4}\right]=-\mathbf{e}_{3},
$$

and hence $\mathfrak{s o l}_{1}^{4^{(1)}}:=\left[\mathfrak{s o l}_{1}^{4},\left.\mathfrak{s o l}\right|_{1} ^{4}\right]=\left\langle\mathbf{e}_{1}, \mathbf{e}_{2}, \mathbf{e}_{3}\right\rangle$ and $\mathfrak{s o l}_{1}^{4(2)}=\mathcal{Z}\left(\mathfrak{s o l} \mathfrak{l}_{1}^{4}\right)=\left\langle\mathbf{e}_{1}\right\rangle$.

Let $\varphi: \mathfrak{s o l}_{1}^{4} \rightarrow \mathfrak{s o l}_{1}^{4}$ be a Lie algebra endomorphism. Since $\varphi$ preserves $\mathfrak{s o r}_{1}^{4^{(1)}}$ and $\mathfrak{s o r}_{1}^{4(2)}$, we have

$$
\varphi\left(\mathbf{e}_{1}\right)=p_{11} \mathbf{e}_{1},
$$




$$
\begin{aligned}
& \varphi\left(\mathbf{e}_{2}\right)=p_{12} \mathbf{e}_{1}+p_{22} \mathbf{e}_{2}+p_{32} \mathbf{e}_{3}, \\
& \varphi\left(\mathbf{e}_{3}\right)=p_{13} \mathbf{e}_{1}+p_{23} \mathbf{e}_{2}+p_{33} \mathbf{e}_{3}, \\
& \varphi\left(\mathbf{e}_{4}\right)=p_{14} \mathbf{e}_{1}+p_{24} \mathbf{e}_{2}+p_{34} \mathbf{e}_{3}+p_{44} \mathbf{e}_{4},
\end{aligned}
$$

for some constants $p_{i j}$. Because $\varphi$ preserves the Lie brackets, it follows that $\varphi$ can be expressed as a matrix of one of the following three forms:

$$
\begin{aligned}
{\left[\begin{array}{cccc}
x y & w x & v y & u \\
0 & x & 0 & v \\
0 & 0 & y & w \\
0 & 0 & 0 & 1
\end{array}\right], \quad\left[\begin{array}{cccc}
-1 & 0 & 0 & 0 \\
0 & 0 & 1 & 0 \\
0 & 1 & 0 & 0 \\
0 & 0 & 0 & -1
\end{array}\right]\left[\begin{array}{cccc}
x y & w x & v y & u \\
0 & x & 0 & v \\
0 & 0 & y & w \\
0 & 0 & 0 & 1
\end{array}\right],\left[\begin{array}{cccc}
0 & 0 & 0 & u \\
0 & 0 & 0 & v \\
0 & 0 & 0 & w \\
0 & 0 & 0 & p
\end{array}\right] } \\
(p \neq \pm 1) .
\end{aligned}
$$

In particular, we have

$$
\operatorname{Aut}_{0}\left(\mathfrak{s o r}_{1}^{4}\right)=\left\{\left[\begin{array}{cccc}
x y & w x & v y & u \\
0 & x & 0 & v \\
0 & 0 & y & w \\
0 & 0 & 0 & 1
\end{array}\right] \mid x, y, u, v, w \in \mathbb{R}, x y \neq 0\right\} .
$$

Let

$$
\tau=\left[\begin{array}{cccc}
-1 & 0 & 0 & 0 \\
0 & 0 & 1 & 0 \\
0 & 1 & 0 & 0 \\
0 & 0 & 0 & -1
\end{array}\right]
$$

The subgroup of $\operatorname{Aut}_{0}\left(\mathfrak{s o r}_{1}^{4}\right)$ with $x, y= \pm 1$ and $u=v=w=0$ is isomorphic to $\left(\mathbb{Z}_{2}\right)^{2}$, which is a maximal compact subgroup. Hence $\operatorname{Aut}\left(\mathfrak{s o l}_{1}^{4}\right)$ has a subgroup generated by $\left(\mathbb{Z}_{2}\right)^{2}$ and $\tau$ as a maximal compact subgroup. This group is isomorphic to the dihedral group $D(4)$ of order 8 .

A connected solvable Lie group $S$ is called of type (R) (or completely solvable) if ad $(X)$ : $\mathfrak{S} \rightarrow \mathfrak{S}$ has only real eigenvalues for each $X \in \mathfrak{S}$. It is known that any Lie group $S$ of type $(\mathrm{R})$ is of type $(\mathrm{E})$, i.e., $\exp : \mathfrak{S} \rightarrow S$ is surjective. Remark that $\mathfrak{s o l} \mathfrak{l}_{1}^{4}$ is a 3-step unimodular and completely solvable (or of type (R)) Lie algebra. Hence the exponential map exp : $\mathfrak{s o l} \mathfrak{l}_{1}^{4} \rightarrow$ $\mathrm{Sol}_{1}^{4}$ is a diffeomorphism with inverse log, and they are given explicitly as follows:

$$
\begin{gathered}
\exp :\left[\begin{array}{lll}
0 & b & a \\
0 & \theta & c \\
0 & 0 & 0
\end{array}\right] \longmapsto\left[\begin{array}{ccc}
1 & \frac{e^{\theta}-1}{\theta} b & a+\frac{e^{\theta}-1-\theta}{\theta^{2}} b c \\
0 & \theta & \frac{e^{\theta}-1}{\theta} c \\
0 & 0 & 1
\end{array}\right], \\
\log :\left[\begin{array}{ccc}
1 & y & x \\
0 & e^{\theta} & z \\
0 & 0 & 1
\end{array}\right] \longmapsto\left[\begin{array}{ccc}
0 & \frac{\theta}{e^{\theta}-1} y & x-\frac{e^{\theta}-1-\theta}{\left(e^{\theta}-1\right)^{2}} y z \\
0 & \theta & \frac{\theta}{e^{\theta}-1} z \\
0 & 0 & 0
\end{array}\right] .
\end{gathered}
$$

Because $\mathrm{Sol}_{1}^{4}$ is simply connected, every Lie group endomorphism is understood as the composition exp $\circ \circ \circ \log$. 
The group $\mathrm{Sol}_{1}^{4}$ has the three-dimensional Heisenberg group $\mathrm{Nil}^{3}(\theta=0)$ as its nilradical. Indeed, the derived group of $\mathrm{Sol}_{1}^{4}$ is $\mathrm{Nil}^{3}$. On the other hand, $\mathrm{Sol}_{1}^{4}$ has the center $\mathcal{Z}\left(\mathrm{Sol}_{1}^{4}\right)=$ $\mathbb{R}(y=z=\theta=0)$, and the quotient turns out to be isomorphic to a three-dimensional solvable Lie group $\mathrm{Sol}^{3}$, see Remark 2.1. That is,

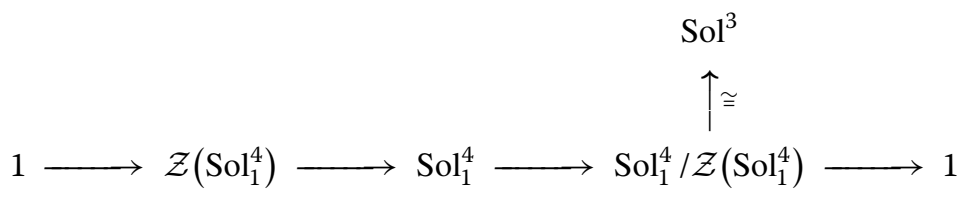

where the isomorphism is given by

$$
\left[\begin{array}{ccc}
1 & y & * \\
0 & e^{\theta} & z \\
0 & 0 & 1
\end{array}\right] \longmapsto\left(\left[\begin{array}{c}
e^{-\theta} y \\
z
\end{array}\right],-\theta\right)
$$

Consequently, $\mathrm{Sol}_{1}^{4}$ fits in the following commutative diagram between short exact sequences ( $c f .[13])$ :

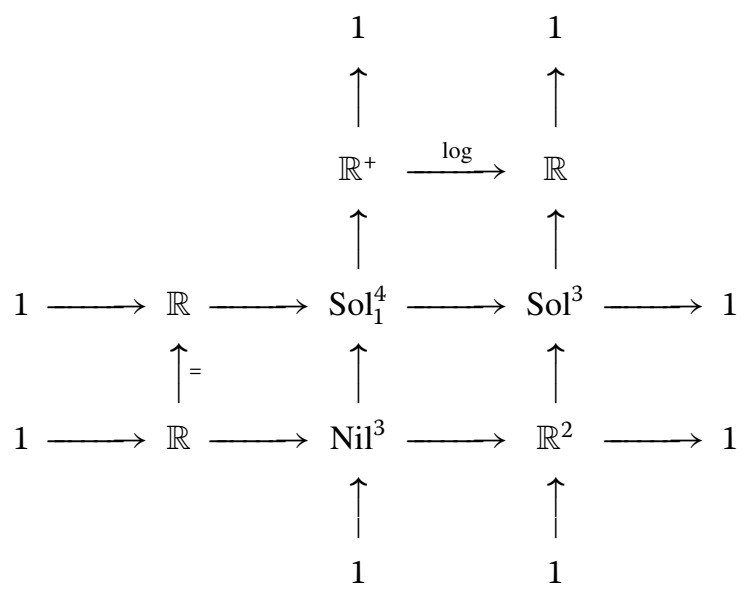

Remark 2.1 Recall that $\mathrm{Sol}^{3}$ is one of the eight geometries in dimension 3. We denote by $\mathbb{R}^{1,1}$ the vector space $\mathbb{R}^{2}$ with the bilinear form

$$
\mathbf{b}(\mathbf{x}, \mathbf{y})=-x_{1} y_{1}+x_{2} y_{2}
$$

Let $\mathbb{E}(1,1)$ denote the group of all isometries of $\mathbb{R}^{1,1}$. Then $\mathbb{E}(1,1)=\mathbb{R}^{2} \rtimes \mathrm{O}(1,1)$, where $\mathrm{O}(1,1)$ denotes the orthogonal group of $\mathbf{b}$ :

$$
\mathrm{O}(1,1)=\left\{\left[\begin{array}{cc}
\alpha & 0 \\
0 & \alpha^{-1}
\end{array}\right] \mid \alpha \neq 0\right\} .
$$

Hence

$$
\mathrm{SE}(1,1)=\mathbb{R}^{2} \rtimes \mathrm{SO}(1,1)=\mathbb{R}^{2} \rtimes\left\{\left[\begin{array}{cc}
e^{\theta} & 0 \\
0 & e^{-\theta}
\end{array}\right] \mid \theta \in \mathbb{R}\right\} .
$$


This shows that $\mathrm{SE}(1,1) \cong \mathbb{R}^{2} \rtimes_{\sigma} \mathbb{R}$, where

$$
\sigma: \mathbb{R} \rightarrow \mathrm{GL}(2, \mathbb{R}), \quad \theta \mapsto\left[\begin{array}{cc}
e^{\theta} & 0 \\
0 & e^{-\theta}
\end{array}\right]
$$

Recall that $\operatorname{Sol}^{3}$ is the group $\mathbb{R}^{2} \rtimes_{\sigma} \mathbb{R}$. We also remark that the map $\operatorname{Sol}_{1}^{4} \rightarrow \operatorname{GL}(3, \mathbb{R})$,

$$
\left[\begin{array}{ccc}
1 & y & * \\
0 & e^{\theta} & z \\
0 & 0 & 1
\end{array}\right] \longmapsto\left[\begin{array}{ccc}
1 & 0 & 0 \\
e^{-\theta} y+z & \cosh (-\theta) & \sinh (-\theta) \\
e^{-\theta} y-z & \sinh (-\theta) & \cosh (-\theta)
\end{array}\right]
$$

is a Lie group homomorphism with kernel $\mathcal{Z}\left(\mathrm{Sol}_{1}^{4}\right)=\mathbb{R}$. Consequently, this induces that $\mathrm{Sol}^{3}$ can be embedded into $\mathrm{GL}(3, \mathbb{R})$ as

$$
\left(\left[\begin{array}{l}
y \\
z
\end{array}\right], \theta\right) \longmapsto\left[\begin{array}{ccc}
1 & 0 & 0 \\
y+z & \cosh \theta & \sinh \theta \\
y-z & \sinh \theta & \cosh \theta
\end{array}\right]
$$

As observed above, $\mathrm{Sol}^{3}$ can be embedded naturally into the affine group $\operatorname{Aff}\left(\mathbb{R}^{2}\right)$ as

$$
\left(\left[\begin{array}{l}
y \\
z
\end{array}\right], \theta\right) \longmapsto\left[\begin{array}{ccc}
e^{\theta} & 0 & y \\
0 & e^{-\theta} & z \\
0 & 0 & 1
\end{array}\right]
$$

\section{The lattices of Sol ${ }_{1}^{4}$}

In this section we study lattices $\Gamma$ of $\mathrm{Sol}_{1}^{4}$ with [12] as our basic reference, and then we study continuous maps on the solvmanifold $\Gamma \backslash \mathrm{Sol}_{1}^{4}$ up to homotopy.

Theorem 3.1 Every lattice $\Gamma$ of $\mathrm{Sol}_{1}^{4}$ can be generated by $\gamma_{0}, \gamma_{1}, \gamma_{2}, \gamma_{3}$ with relations

$$
\begin{aligned}
& {\left[\gamma_{1}, \gamma_{2}\right]=\gamma_{3}^{k}, \quad\left[\gamma_{1}, \gamma_{3}\right]=\left[\gamma_{2}, \gamma_{3}\right]=1,} \\
& \gamma_{0} \gamma_{1} \gamma_{0}^{-1}=\gamma_{1}^{n_{11}} \gamma_{2}^{n_{21}} \gamma_{3}^{p_{1}}, \quad \gamma_{0} \gamma_{2} \gamma_{0}^{-1}=\gamma_{1}^{n_{12}} \gamma_{2}^{n_{22}} \gamma_{3}^{p_{2}}, \quad \gamma_{0} \gamma_{3} \gamma_{0}^{-1}=\gamma_{3}
\end{aligned}
$$

for some integers $k, p_{1}, p_{2}$ with $k \neq 0$ and $N=\left[n_{i j}\right] \in \operatorname{SL}(2, \mathbb{Z})$ with trace $>2$.

Notation We denote such a lattice of $\operatorname{Sol}_{1}^{4}$ by $\Gamma_{k, N, \mathbf{p}}$.

Proof Consider the derived series of $\mathrm{Sol}_{1}^{4}: \mathrm{Sol}_{1}^{4} \supset \mathrm{Nil}^{3} \supset \mathcal{Z}\left(\mathrm{Nil}^{3}\right)$. Taking intersections with $\Gamma$, we obtain

$$
\Gamma=\Gamma_{0} \supset \Gamma_{1} \supset \Gamma_{2},
$$


where $\Gamma_{1}$ is a lattice of $\mathrm{Nil}^{3}$. From the commutative diagram (S), we obtain a commutative diagram between lattices

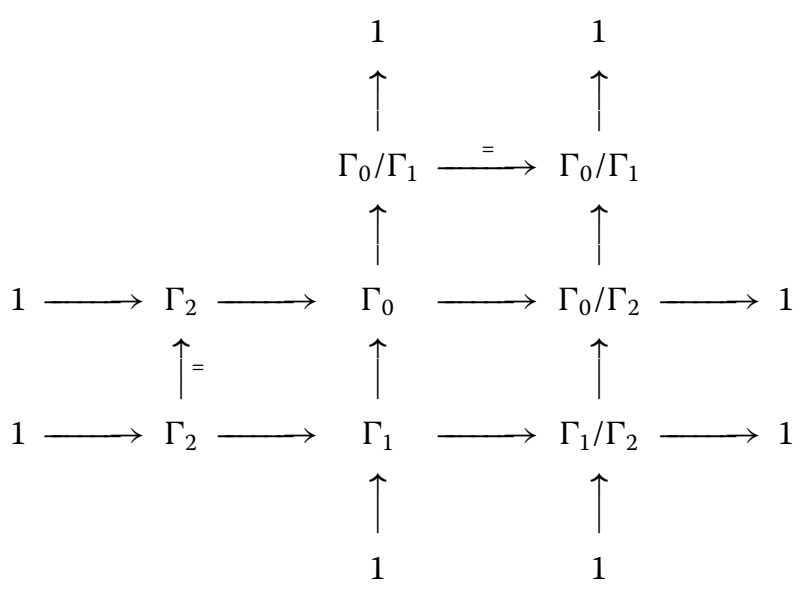

Remark that the bottom exact sequence comes from the short exact sequence $1 \rightarrow \mathbb{R} \rightarrow$ $\mathrm{Nil}^{3} \rightarrow \mathbb{R}^{2} \rightarrow 1$. Then it is well known, for example, in [14] that such $\Gamma_{1}$ is generated by $\gamma_{1}$, $\gamma_{2}, \gamma_{3}$ satisfying the relations

$$
\left[\gamma_{1}, \gamma_{3}\right]=\left[\gamma_{2}, \gamma_{3}\right]=1, \quad\left[\gamma_{1}, \gamma_{2}\right]=\gamma_{3}^{k}
$$

for some nonzero integer $k$. In particular, $\gamma_{3}$ is a generator of $\Gamma_{2} \cong \mathbb{Z}$ and $\tilde{\gamma}_{1}, \tilde{\gamma}_{2}$ generate $\Gamma_{1} / \Gamma_{2} \cong \mathbb{Z}^{2}$. Now, from the middle vertical, we can choose $\gamma_{0} \in \Gamma_{0}$ so that $\left\{\gamma_{0}, \ldots, \gamma_{3}\right\}$ generates $\Gamma_{0}$. We denote by $\tilde{\gamma}_{0}$ and $\tilde{\tilde{\gamma}}_{0}$ the images of $\gamma_{0}$ under the projections $\Gamma_{0} \rightarrow \Gamma_{0} / \Gamma_{2}$ and $\Gamma_{0} \rightarrow \Gamma_{0} / \Gamma_{1}$, respectively. Remark also that $\left\{\tilde{\gamma}_{0}, \tilde{\gamma}_{1}, \tilde{\gamma}_{2}\right\}$ is a generator set of $\Gamma_{0} / \Gamma_{2}$, which is a lattice of $\operatorname{Sol}^{3}$. Because $\left\{\tilde{\gamma}_{1}, \tilde{\gamma}_{2}\right\}$ generates $\Gamma_{1} / \Gamma_{2} \cong \mathbb{Z}^{2}$ and $\tilde{\tilde{\gamma}}_{0}$ generates $\Gamma_{0} / \Gamma_{1} \cong$ $\mathbb{Z}$, we must have

$$
\left[\tilde{\gamma}_{1}, \tilde{\gamma}_{2}\right]=1, \quad \tilde{\gamma_{0}} \tilde{\gamma}_{j} \tilde{\gamma}_{0}^{-1}=\tilde{\gamma}_{1}^{n_{1 j}} \tilde{\gamma}_{2}^{n_{2 j}}
$$

for some integers $n_{i j}$. Let $N=\left[n_{i j}\right]$. Then it can be seen that $N \in \mathrm{SL}(2, \mathbb{Z})$ with trace $>2$. For details about lattices of $\mathrm{Sol}^{3}$, we refer to $[10,15]$. On the other hand, the conjugation by $\gamma_{0}$ induces an automorphism on $\Gamma_{1}$. Because this automorphism must preserve the relation $\left[\gamma_{1}, \gamma_{2}\right]=\gamma_{3}^{k}$, it follows that $\gamma_{0} \gamma_{3} \gamma_{0}^{-1}=\gamma_{3}^{\operatorname{det}(N)}=\gamma_{3}$. Consequently, the theorem is proved.

Now we will study an embedding of an abstract group $\Gamma_{k, N, \mathbf{p}}$ into $\mathrm{Sol}_{1}^{4}$ as a lattice. Let $N$ be a $2 \times 2$ hyperbolic integer matrix with trace $>2$. Then $N$ has two distinct irrational eigenvalues $e^{\theta_{0}}$ and $e^{-\theta_{0}}$ with corresponding eigenvectors $\left(y_{1}, z_{1}\right)$ and $\left(y_{2}, z_{2}\right)$. This means that $N Q=Q \Theta$, where $Q$ is the matrix with columns $\left(y_{1}, z_{1}\right)^{t}$ and $\left(y_{2}, z_{2}\right)^{t}$ and $\Theta$ is the diagonal matrix with entries $e^{\theta_{0}}$ and $e^{-\theta_{0}}$. From the identity $N Q=Q \Theta$, we can now check that the assignment

$$
\tilde{\gamma}_{0} \mapsto\left[\begin{array}{ccc}
1 & 0 & 0 \\
0 & e^{-\theta_{0}} & 0 \\
0 & 0 & 1
\end{array}\right], \quad \tilde{\gamma}_{j} \mapsto\left[\begin{array}{ccc}
1 & y_{j} & 0 \\
0 & 1 & z_{j} \\
0 & 0 & 1
\end{array}\right]
$$


realizes $\Gamma_{0} / \Gamma_{2}$ as a lattice of $\operatorname{Sol}^{3}=\operatorname{Sol}_{1}^{4} / \mathcal{Z}\left(\operatorname{Sol}_{1}^{4}\right)$. Thus, by lifting $\tilde{\gamma}_{0}, \tilde{\gamma}_{1}, \tilde{\gamma}_{2} \in \Gamma_{0} / \Gamma_{2}$ in $\Gamma_{0}$, we have

$$
\gamma_{0} \mapsto\left[\begin{array}{ccc}
1 & 0 & x_{0} \\
0 & e^{-\theta_{0}} & 0 \\
0 & 0 & 1
\end{array}\right], \quad \gamma_{j} \mapsto\left[\begin{array}{ccc}
1 & y_{j} & x_{j} \\
0 & 1 & z_{j} \\
0 & 0 & 1
\end{array}\right], \quad \gamma_{3} \mapsto\left[\begin{array}{ccc}
1 & 0 & x_{3} \\
0 & 1 & 0 \\
0 & 0 & 1
\end{array}\right]
$$

for some $x_{0}, x_{1}, x_{2}, x_{3} \in \mathbb{R}$. The relation $\left[\gamma_{1}, \gamma_{2}\right]=\gamma_{3}^{k}$ yields $x_{3}=\left(y_{1} z_{2}-y_{2} z_{1}\right) / k$, and then the relation $\gamma_{0} \gamma_{j} \gamma_{0}^{-1}=\gamma_{1}^{n_{1 j}} \gamma_{2}^{n_{2 j}} \gamma_{3}^{p_{j}}$ yields that $x_{j}=\left(y_{1} z_{2}-y_{2} z_{1}\right) p_{j} / k$. We can choose $x_{0}$ arbitrarily. Therefore, this gives an embedding $\Gamma$ into $\mathrm{Sol}_{1}^{4}$ as a lattice.

In the theorem below, we study the homomorphisms on any lattice of $\mathrm{Sol}_{1}^{4}$.

Theorem 3.2 Let

$$
\Gamma_{k, N, \mathbf{p}}=\left(\begin{array}{l|l}
\gamma_{0}, \gamma_{1}, \gamma_{2}, \gamma_{3} & \begin{array}{c}
{\left[\gamma_{1}, \gamma_{2}\right]=\gamma_{3}^{k},\left[\gamma_{0}, \gamma_{3}\right]=\left[\gamma_{1}, \gamma_{3}\right]=\left[\gamma_{2}, \gamma_{3}\right]=1,} \\
\gamma_{0} \gamma_{1} \gamma_{0}^{-1}=\gamma_{1}^{n_{11}} \gamma_{2}^{n_{21}} \gamma_{3}^{p_{1}}, \gamma_{0} \gamma_{2} \gamma_{0}^{-1}=\gamma_{1}^{n_{12}} \gamma_{2}^{n_{22}} \gamma_{3}^{p_{2}}
\end{array}
\end{array}\right\}
$$

be a lattice of $\mathrm{Sol}_{1}^{4}$. Then any homomorphism $\phi$ on $\Gamma_{k, N, \mathbf{p}}$ is either one of the following forms:

$$
\begin{aligned}
\text { Type (I) } \phi\left(\gamma_{0}\right) & =\gamma_{0} \gamma_{1}^{r_{1}} \gamma_{2}^{r_{2}} \gamma_{3}^{q_{0}}, \phi\left(\gamma_{1}\right)=\gamma_{1}^{\mu} \gamma_{2}^{\frac{n_{21} 1}{n_{12}} v} \gamma_{3}^{q_{1}}, \phi\left(\gamma_{2}\right)=\gamma_{1}^{v} \gamma_{2}^{\mu+\frac{n_{22}-n_{11}}{n_{12}} v} \gamma_{3}^{q_{2}}, \\
\phi\left(\gamma_{3}\right) & =\gamma_{3}^{\mu\left(\mu+\frac{n_{22}-n_{11}}{n_{12}} v\right)-\frac{n_{21}}{n_{12}} v^{2}} ; \\
\text { Type (II) } \phi\left(\gamma_{0}\right) & =\gamma_{0}^{-1} \gamma_{1}^{r_{1}} \gamma_{2}^{r_{2}} \gamma_{3}^{q_{0}}, \phi\left(\gamma_{1}\right)=\gamma_{1}^{-\mu} \gamma_{2}^{v} \gamma_{3}^{q_{1}}, \phi\left(\gamma_{2}\right)=\gamma_{1}^{\frac{n_{11}-n_{22}}{n_{21}} \mu-\frac{n_{12} v}{n_{21} v}} \gamma_{2}^{\mu} \gamma_{3}^{q_{2},} \\
\phi\left(\gamma_{3}\right) & =\gamma_{3}^{-\mu^{2}-\left(\frac{n_{11}-n_{22}}{n_{21}} \mu-\frac{n_{12}}{n_{21}} v\right) v} ;
\end{aligned}
$$

Type (III) $\phi\left(\gamma_{0}\right)=\gamma_{0}^{m} \gamma_{1}^{r_{1}} \gamma_{2}^{r_{2}} \gamma_{3}^{q_{0}}, \phi\left(\gamma_{1}\right)=\gamma_{3}^{q_{1}}, \phi\left(\gamma_{2}\right)=\gamma_{3}^{q_{2}}, \phi\left(\gamma_{3}\right)=1$ with $m \neq \pm 1$.

Proof For simplicity, we write $\Gamma=\Gamma_{k, N, \mathbf{p}}$. Let $\phi: \Gamma \rightarrow \Gamma$ be any homomorphism. Consider the derived series of $\mathrm{Sol}_{1}^{4}: \mathrm{Sol}_{1}^{4} \supset \mathrm{Nil}^{3} \supset \mathcal{Z}\left(\mathrm{Nil}^{3}\right)$, and its associated sequence $\Gamma=\Gamma_{0} \supset$ $\Gamma_{1} \supset \Gamma_{2}$ obtained by taking intersections with $\Gamma$. Note that $\Gamma_{1}=\left\langle\gamma_{1}, \gamma_{2}, \gamma_{3}\right\rangle$ and $\Gamma_{2}=\left\langle\gamma_{3}\right\rangle$. Because $\mathrm{Sol}_{1}^{4}$ is of type (R), the homomorphism $\phi$ on $\Gamma$ extends uniquely to a Lie group homomorphism $\Phi$ on $\mathrm{Sol}_{1}^{4}$, see, for example, [16], Corollary 8.3, or [17, 18]. Now, $\Phi$ preserves the derived series of $\mathrm{Sol}_{1}^{4}$, and so $\phi$ preserves the associated sequence. In particular, $\left.\phi\right|_{\Gamma_{1}}$ is a homomorphism on the lattice $\Gamma_{1}$ of $\mathrm{Nil}^{3}$. Since $\Gamma_{1}=\left\langle\gamma_{1}, \gamma_{2}, \gamma_{2} \mid\left[\gamma_{1}, \gamma_{2}\right]=\gamma_{3}^{k}\right\rangle$, it follows that

$$
\begin{aligned}
& \phi\left(\gamma_{0}\right)=\gamma_{0}^{m} \gamma_{1}^{r_{1}} \gamma_{2}^{r_{2}} \gamma_{3}^{q_{0}}, \\
& \phi\left(\gamma_{1}\right)=\gamma_{1}^{m_{11}} \gamma_{2}^{m_{21}} \gamma_{3}^{q_{1}}, \\
& \phi\left(\gamma_{2}\right)=\gamma_{1}^{m_{12}} \gamma_{2}^{m_{22}} \gamma_{3}^{q_{2}}, \\
& \phi\left(\gamma_{3}\right)=\gamma_{3}^{m_{11} m_{22}-m_{12} m_{21},}
\end{aligned}
$$

where $m_{i j}, r_{i}, q_{j} \in \mathbb{Z}$, see [8], Lemma 2.1, for $\left.\phi\right|_{\Gamma_{1}}$. Further, $\phi$ induces a homomorphism $\tilde{\phi}$ on the lattice $\Gamma_{0} / \Gamma_{2}$ of $\mathrm{Sol}^{3}$ so that

$$
\tilde{\phi}\left(\tilde{\gamma}_{0}\right)=\tilde{\gamma}_{0}^{m} \tilde{\gamma}_{1}^{r_{1}} \tilde{\gamma}_{2}^{r_{2}}, \quad \tilde{\phi}\left(\tilde{\gamma}_{1}\right)=\tilde{\gamma}_{1}^{m_{11}} \tilde{\gamma}_{2}^{m_{21}}, \quad \tilde{\phi}\left(\tilde{\gamma}_{2}\right)=\tilde{\gamma}_{1}^{m_{12}} \tilde{\gamma}_{2}^{m_{22}} .
$$


Note that if we put $M=\left[m_{i j}\right]$, then the relations in the lattice

$$
\Gamma_{0} / \Gamma_{2}=\left\langle\tilde{\gamma}_{0} \cdot \tilde{\gamma}_{1}, \tilde{\gamma}_{2} \mid\left[\tilde{\gamma}_{1}, \tilde{\gamma}_{2}\right]=1, \tilde{\gamma}_{0} \tilde{\gamma}_{1} \tilde{\gamma}_{0}^{-1}=\tilde{\gamma}_{1}^{n_{11}} \tilde{\gamma}_{2}^{n_{21}}, \tilde{\gamma}_{0} \tilde{\gamma}_{2} \tilde{\gamma}_{0}^{-1}=\tilde{\gamma}_{1}^{n_{12}} \tilde{\gamma}_{2}^{n_{22}}\right\rangle
$$

induce the identity $N^{m} M=M N$. Recall from the observation earlier that there exists a matrix $Q$ such that $Q^{-1} N Q=\Theta$. Hence $\Theta^{m}\left(Q^{-1} M Q\right)=\left(Q^{-1} M Q\right) \Theta$. Because $\Theta$ is a diagonal matrix of determinant 1 , it follows that when $m=1, Q^{-1} M Q$ is a diagonal matrix; when $m=-1, Q^{-1} M Q$ is an off-diagonal matrix; when $m \neq \pm 1, Q^{-1} M Q$ and hence $M$ is a zero matrix. These yield the three possibilities for $\tilde{\phi}$. For details, we refer to [10], Theorem 2.4. All these observations deduce the proof.

Remark 3.3 (Homomorphisms on $\Gamma$ up to conjugacy) Let $\phi$ be any homomorphism on $\Gamma=\Gamma_{k, N, \mathbf{p}}$ given as in Theorem 3.2. We will observe the effect of $\phi$ under the conjugation $\mu(\gamma)$ by an element $\gamma \in \Gamma$. Assume

$$
\begin{array}{ll}
\phi\left(\gamma_{0}\right)=\gamma_{0}^{m} \gamma_{1}^{r_{1}} \gamma_{2}^{r_{2}} \gamma_{3}^{q_{0}}, & \phi\left(\gamma_{1}\right)=\gamma_{1}^{m_{11}} \gamma_{2}^{m_{21}} \gamma_{3}^{q_{1}}, \\
\phi\left(\gamma_{2}\right)=\gamma_{1}^{m_{12}} \gamma_{2}^{m_{22}} \gamma_{3}^{q_{2}}, & \phi\left(\gamma_{3}\right)=\gamma_{3}^{m_{11} m_{22}-m_{12} m_{21}} .
\end{array}
$$

For any element $\gamma=\gamma_{0}^{\ell} \gamma_{1}^{s} \gamma_{2}^{t} \gamma_{3}^{q}$ in $\Gamma$, we have

$$
\begin{array}{ll}
\mu(\gamma) \circ \phi\left(\gamma_{0}\right)=\gamma_{0}^{m^{\prime}} \gamma_{1}^{r_{1}^{\prime}} \gamma_{2}^{r_{2}^{\prime}} \gamma_{3}^{q_{0}^{\prime}}, & \mu(\gamma) \circ \phi\left(\gamma_{1}\right)=\gamma_{1}^{m_{11}^{\prime}} \gamma_{2}^{m_{21}^{\prime}} \gamma_{3}^{q_{1}^{\prime}}, \\
\mu(\gamma) \circ \phi\left(\gamma_{2}\right)=\gamma_{1}^{m_{12}^{\prime}} \gamma_{2}^{m_{22}^{\prime}} \gamma_{3}^{q_{2}^{\prime}}, & \mu(\gamma) \circ \phi\left(\gamma_{3}\right)=\gamma_{3}^{m_{11} m_{22}-m_{12} m_{21}} .
\end{array}
$$

We will first have a look at $\mu(\gamma) \circ \phi \bmod \Gamma_{2}$, i.e., $\mu(\tilde{\gamma}) \circ \tilde{\phi}$ on the lattice $\Gamma_{0} / \Gamma_{2}$. Since in $\bmod \Gamma_{2},\left[\gamma_{1}, \gamma_{2}\right] \equiv 1$ and the conjugation by $\gamma_{0}$ is the multiplication by $N$ on $\mathbb{Z}^{2} \equiv\left\langle\gamma_{1}, \gamma_{2}\right\rangle$, we can show easily that

$$
m^{\prime}=m, \quad\left[\begin{array}{l}
m_{1 i}^{\prime} \\
m_{2 i}^{\prime}
\end{array}\right]=N^{\ell}\left[\begin{array}{l}
m_{1 i} \\
m_{2 i}
\end{array}\right], \quad\left[\begin{array}{l}
r_{1}^{\prime} \\
r_{2}^{\prime}
\end{array}\right]=N^{\ell}\left(\left(N^{-m}-I\right)\left[\begin{array}{l}
s \\
t
\end{array}\right]+\left[\begin{array}{l}
r_{1} \\
r_{2}
\end{array}\right]\right) .
$$

\section{Continuous maps on solvmanifolds $\Gamma \backslash$ Sol $_{1}^{4}$}

Let $f: \Gamma \backslash \operatorname{Sol}_{1}^{4} \rightarrow \Gamma \backslash \mathrm{Sol}_{1}^{4}$ be a continuous map. Fixing a lift $\tilde{f}$ of $f$ on the universal cover $\mathrm{Sol}_{1}^{4}, f$ defines a homomorphism $\varphi$ on the group of covering transformations $\Gamma$, namely,

$$
\varphi(\gamma) \circ \tilde{f}=\tilde{f} \circ \gamma, \quad \forall \gamma \in \Gamma .
$$

Thus $\varphi$ is a homomorphism in Theorem 3.2. This homomorphism extends uniquely to a Lie group homomorphism $\Phi$ on $\mathrm{Sol}_{1}^{4}$. This implies that $\Phi$ restricts to a map $\Phi_{\Gamma}$ on the quotient space $\Gamma \backslash \operatorname{Sol}_{1}^{4}$ which induces the same homomorphism $\varphi$ on $\Gamma$. Further, since $\Gamma \backslash \mathrm{Sol}_{1}^{4}$ is aspherical, $\Phi_{\Gamma}$ is homotopic to $f$. Remark also that another choice of a lift of $f$ results in a new homomorphism on $\Gamma$ and on $\mathrm{Sol}_{1}^{4}$ which differ by the conjugation by an element of $\Gamma$.

Since the invariants that we are going to deal with are all homotopy invariants, we will assume in what follows that every continuous map on $\Gamma \backslash \mathrm{Sol}_{1}^{4}$ is induced by a Lie group homomorphism $\Phi$ on $\mathrm{Sol}_{1}^{4}$ preserving $\Gamma$. 
Let $\Phi$ be a Lie group homomorphism on $\mathrm{Sol}_{1}^{4}$. Then we obtain the following commutative diagram:

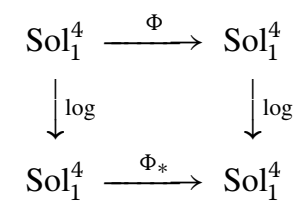

where $\Phi_{*}$ is the differential of $\Phi$. Assume that $\Phi$ restricts to a homomorphism $\varphi$ on $\Gamma$. Recall that $\Phi$ preserves the derived series of $\mathrm{Sol}_{1}^{4}, \mathrm{Sol}_{1}^{4} \supset \mathrm{Nil}^{3} \supset \mathcal{Z}\left(\mathrm{Sol}_{1}^{4}\right)$, and its associated sequence $\Gamma \supset \Gamma_{1} \supset \Gamma_{2}$. Then we could choose a set of generators $\left\{\gamma_{0}, \gamma_{1}, \gamma_{2}, \gamma_{3}\right\}$ so that $\gamma_{1}, \gamma_{2} \in \mathrm{Nil}^{3}$ and $\gamma_{3} \in \mathcal{Z}\left(\operatorname{Sol}_{1}^{4}\right)$. Furthermore, the image of $\gamma_{0}$ under the projection $\Gamma \rightarrow$ $\Gamma / \Gamma_{1}$ generates $\Gamma / \Gamma_{1} \cong \mathbb{Z}$, the images of $\gamma_{1}, \gamma_{2}$ under the projection $\Gamma_{1} \rightarrow \Gamma_{1} / \Gamma_{2}$ generate $\Gamma_{1} / \Gamma_{2} \cong \mathbb{Z}^{2}$, and $\gamma_{3}$ generates $\Gamma_{3} \cong \mathbb{Z}$. Therefore, $\left\{\log \gamma_{0}, \log \gamma_{1}, \log \gamma_{2}, \log \gamma_{3}\right\}$ forms a linear ordered basis of $\mathrm{Sol}_{1}^{4}$ with respect to which $\Phi_{*}$ can be expressed as a matrix of the form

$$
\Phi_{*}=\left[\begin{array}{c:c:c}
\varphi_{0} & 0 & \vdots \\
\hdashline * & \cdots & \cdots \\
* & \varphi_{1} & 0 \\
\hdashline \ldots & \cdots & \ldots \\
* & * & \varphi_{2}
\end{array}\right],
$$

where $\varphi_{0}, \varphi_{2}$ are integers and $\varphi_{1}$ is a $2 \times 2$ integer matrix. When $f$ is a map on $\Gamma \backslash \operatorname{Sol}_{1}^{4}$, which is homotopic to a map induced by $\Phi$, we say that $\Phi_{*}$ is a linearization of $f$. Because in this paper we are only concerned with the eigenvalues of $\Phi_{*}$, we shall denote by $\Phi_{*}$ the diagonal block integer matrix $\operatorname{diag}\left\{\varphi_{0}, \varphi_{1}, \varphi_{2}\right\}$.

The commutative diagram

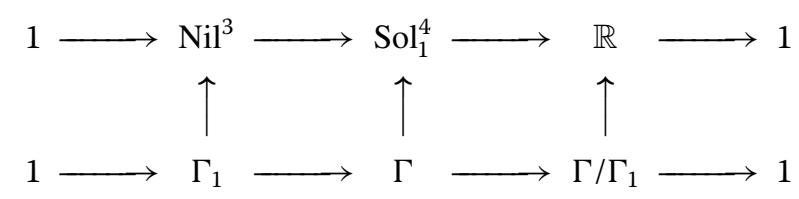

gives rise to a fibration structure on $\Gamma \backslash \mathrm{Sol}_{1}^{4}$, called the Mostow fibration,

$$
\Gamma_{1} \backslash \mathrm{Nil}^{3} \longrightarrow \Gamma \backslash \mathrm{Sol}_{1}^{4} \longrightarrow \mathbb{Z} \backslash \mathbb{R}
$$

over the circle base with nilmanifold fiber. We remark that any map $f$ on $\Gamma \backslash \operatorname{Sol}_{1}^{4}$ induced by a Lie group homomorphism of $\mathrm{Sol}_{1}^{4}$ is a fibration map with respect to the above bundle structure. Indeed, such a Lie group homomorphism $\Phi$ on $\operatorname{Sol}_{1}^{4}$ induces maps $f, f^{\prime}$ and $f_{0}$ so that the diagram is commutative

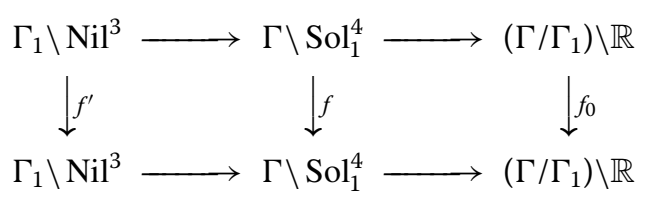

Thus, $\varphi_{0}$ is the degree of the induced $\operatorname{map} f_{0}$ on the base space $\mathbb{Z} \backslash \mathbb{R}$, and

$$
\Phi_{*}^{\prime}=\left[\begin{array}{c:c}
\varphi_{1} & 0 \\
\hdashline * & \ldots \\
\hdashline * & \varphi_{2}
\end{array}\right]
$$


is a linearization of $f^{\prime}$, see [8] for details. In particular, $\varphi_{2}=\operatorname{det} \varphi_{1}$ which was observed already in Theorem 3.2. Furthermore, we also have the following commutative diagram induced by $\Phi$ :

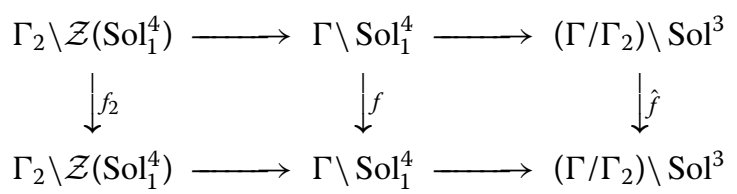

Thus,

$$
\hat{\Phi}_{*}=\left[\begin{array}{c:c}
\varphi_{0} & 0 \\
\hdashline * & \ldots \\
\hdashline \varphi_{1}
\end{array}\right]
$$

is a linearization of $\hat{f}$, see [10] for details. In all, we have

$$
\Phi_{*}=\left[\begin{array}{c:c:c}
\varphi_{0} & 0 & 0 \\
\hdashline * & \cdots & 0 \\
* & \varphi_{1} & 0 \\
\hdashline * & * & \ldots . \\
\hdashline * & * & \varphi_{2}
\end{array}\right]=\left[\begin{array}{c:c}
\varphi_{0} & 0 \\
\hdashline * & \ldots \\
* & \Phi_{*}^{\prime}
\end{array}\right]=\left[\begin{array}{c:c}
\hat{\Phi}_{*} & 0 \\
\hdashline * & \varphi_{2}
\end{array}\right] .
$$

From Theorem 3.2, we immediately obtain the following.

Proposition 4.1 Let $f: \Gamma \backslash \operatorname{Sol}_{1}^{4} \rightarrow \Gamma \backslash \operatorname{Sol}_{1}^{4}$ be a continuous map where $\Gamma=\Gamma_{k, N, \mathbf{p}}$. Then a linearization off is an integer matrix of one of the following:

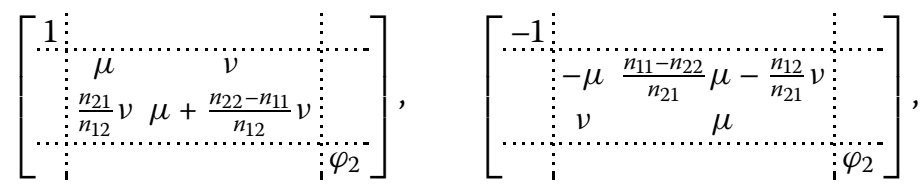

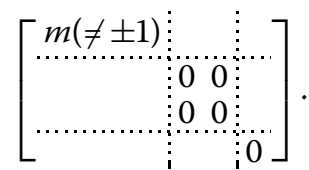

Each linearization is respectively conjugate to

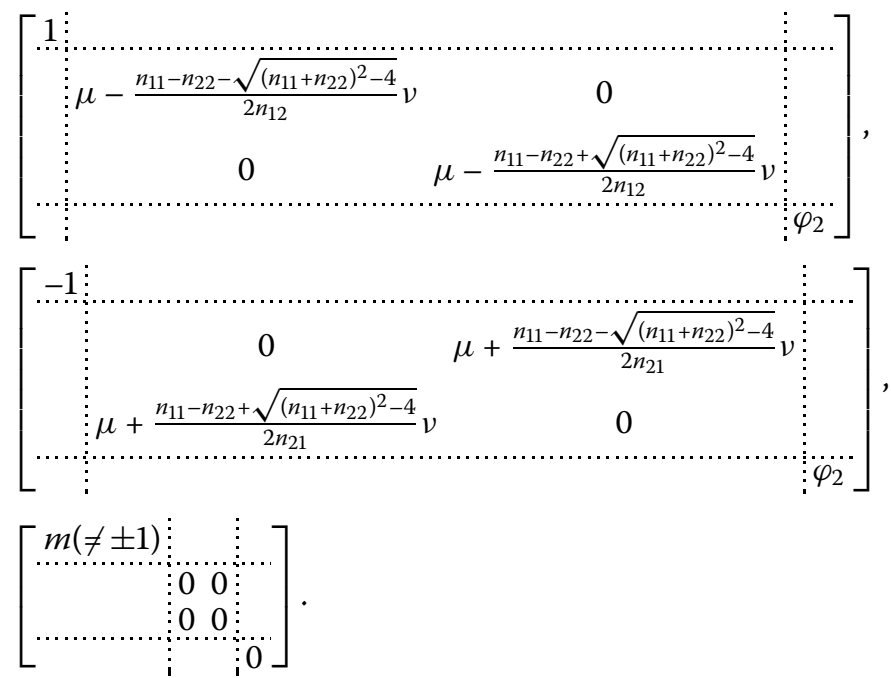


Proof The first part follows from Theorem 3.2. Taking conjugation $Q^{-1}(\cdot) Q$ by the matrix

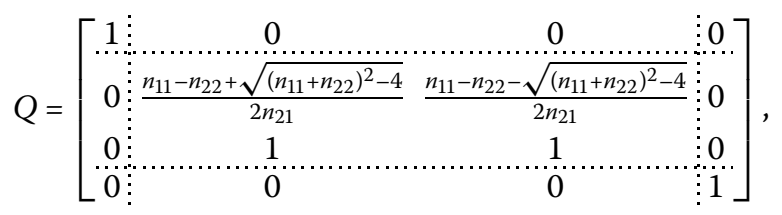

we obtain the second part of our assertion. Notice that the middle block of $Q$ consists of eigenvectors of $N$.

Note that homomorphisms of distinct types are not conjugate to each other. We shall say a map on $\Gamma \backslash \mathrm{Sol}_{1}^{4}$ is of type (I), (II) or (III) according to its homomorphism on $\Gamma$.

Corollary 4.2 Each linearization of a continuous map on $\Gamma \backslash \mathrm{Sol}_{1}^{4}$ is conjugate to one of the following:

(I)

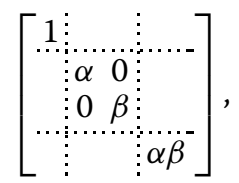

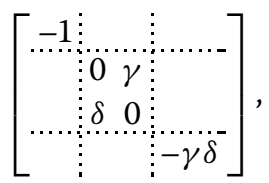

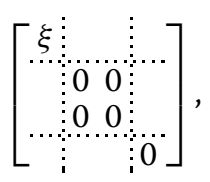

where $\alpha+\beta, \alpha \beta, \gamma \delta, \xi \in \mathbb{Z}$ with $\xi \neq \pm 1$. Moreover, $\alpha=0$ if and only if $\beta=0$, and $\gamma=0$ if and only if $\delta=0$.

Proof Since $\operatorname{tr} N=n_{11}+n_{12}>2$, the number $\sqrt{\left(n_{11}+n_{22}\right)^{2}-4}$ must be irrational. If $\alpha=$ $\mu-\frac{n_{11}-n_{22}-\sqrt{\left(n_{11}+n_{22}\right)^{2}-4}}{2 n_{12}} v=0$, then $v$ must be zero and hence $\mu=0$ because $\mu, v, n_{11}, n_{12}$, $n_{22}$ are all integers. It follows that $\beta=\alpha=0$. The converse is the same. Similarly, we can show that $\gamma=0$ if and only if $\delta=0$.

Remark 4.3 If $f$ is of type (II), then $f^{2}$ is of type (I).

Proposition 4.4 Let $f$ and $f^{\prime}$ be two continuous maps on the solvmanifold $\Gamma_{k, N, \mathbf{p}} \backslash \mathrm{Sol}_{1}^{4}$ with linearizations $\Phi_{*}=\operatorname{diag}\left\{\varphi_{0}, \varphi_{1}, \varphi_{2}\right\}$ and $\Phi_{*}^{\prime}=\operatorname{diag}\left\{\varphi_{0}^{\prime}, \varphi_{1}^{\prime}, \varphi_{2}^{\prime}\right\}$, respectively. Iff and $f^{\prime}$ are homotopic, then

$$
\varphi_{0}^{\prime}=\varphi_{0}, \quad \varphi_{1}^{\prime}=N^{\ell} \varphi_{1}, \quad \varphi_{2}^{\prime}=\varphi_{2}
$$

for some $\ell$.

Proof As it was mentioned earlier, we may assume that $f, f^{\prime}$ are induced respectively by Lie group homomorphisms $\Phi, \Phi^{\prime}: \mathrm{Sol}_{1}^{4} \rightarrow \mathrm{Sol}_{1}^{4}$, both of which restrict to homomorphisms $\varphi, \varphi^{\prime}: \Gamma_{k, N, \mathbf{p}} \rightarrow \Gamma_{k, N, \mathbf{p}}$. Because $f \simeq f^{\prime}, \varphi$ and $\varphi^{\prime}$ differ by the conjugation by an element of $\Gamma_{k, N, \mathbf{p}}$. Now our assertion follows from Remark 3.3.

According to this result, $\operatorname{det} \Phi_{*}$ and $\varphi_{0}, \varphi_{2}$ are all homotopy invariants. A map becomes of type (I), (II) or (III) according to what the value of $\varphi_{0}$ is $1,-1$ or the others. It should be noticed that the $\varphi_{1}$ is not a homotopy invariant. 


\section{Lefschtez numbers and Nielsen type numbers}

Recall that $\operatorname{Sol}_{1}^{4}$ is of type (R). Let $f$ be a map on the special solvmanifold $\Gamma \backslash \operatorname{Sol}_{1}^{4}$ of type (R) with $\Gamma=\Gamma_{k, N, \mathbf{p}}$. Then we may assume that $f$ is induced by a Lie group homomorphism $\Phi$. Consider a linearization $\Phi_{*}=\operatorname{diag}\left\{\varphi_{0}, \varphi_{1}, \varphi_{2}\right\}$ of $f$. Then the main result, Theorem 3.1, of [19] implies that $L(f)=\operatorname{det}\left(I-\Phi_{*}\right)$. By [20], Theorem 2.1, we also have $N(f)=|L(f)|$. See also the main result, Theorem 3.1, of [3].

Next we consider the iterations $f^{n}$ of $f$. Since $\Phi$ induces a map homotopic to $f, \Phi^{n}$ induces a map homotopic to $f^{n}$. Therefore, $\Phi_{*}^{n}=\operatorname{diag}\left\{\varphi_{0}^{n}, \varphi_{1}^{n}, \varphi_{2}^{n}\right\}$ is a linearization of $f^{n}$, and so we have

$$
L\left(f^{n}\right)=\operatorname{det}\left(I-\Phi_{*}^{n}\right)=\left(1-\varphi_{0}^{n}\right) \operatorname{det}\left(I-\varphi_{1}^{n}\right)\left(1-\varphi_{2}^{n}\right) .
$$

Proposition 5.1 Let $f: \Gamma \backslash \operatorname{Sol}_{1}^{4} \rightarrow \Gamma \backslash \mathrm{Sol}_{1}^{4}$ be any continuous map on the solvmanifold $\Gamma \backslash \operatorname{Sol}_{1}^{4}$ with linearization $\Phi_{*}=\operatorname{diag}\left\{\varphi_{0}, \varphi_{1}, \varphi_{2}\right\}$. Then, for all positive integers $n$,

$$
L\left(f^{n}\right)= \begin{cases}0 & \text { when } f \text { is of type }(\mathrm{I}), \\ \left(1-(-1)^{n}\right)\left(1-\varphi_{2}^{2 n}\right) & \text { when } f \text { is of type }(\mathrm{II}), \\ 1-\varphi_{0}^{n} & \text { when } f \text { is of type }(\mathrm{III}),\end{cases}
$$

and $N\left(f^{n}\right)=\left|L\left(f^{n}\right)\right|$.

Proof If $f$ is of type (I), then $\varphi_{0}=\varphi_{0}^{n}=1$ and hence $L\left(f^{n}\right)=0$. If $f$ is of type (III), then $\varphi_{1}=\varphi_{1}^{n}=0$ and $\varphi_{2}=\varphi_{2}^{n}=0$, and hence $L\left(f^{n}\right)=1-\varphi_{0}^{n}$.

Assume that $f$ is of type (II). By Corollary 4.2, we may assume that

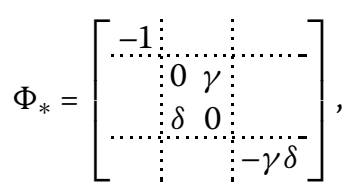

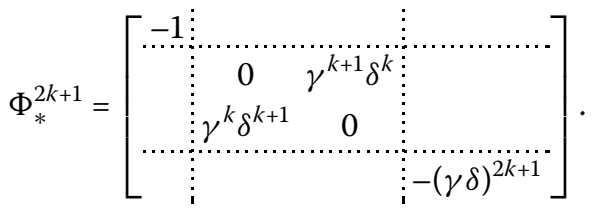

Hence

$$
\begin{aligned}
L\left(f^{2 k+1}\right) & =\operatorname{det}\left(I-\Phi_{*}^{2 k+1}\right)=2\left(1+\operatorname{det} \varphi_{1}^{2 k+1}\right)\left(1-\varphi_{2}^{2 k+1}\right) \\
& =2\left(1-(\gamma \delta)^{2 k+1}\right)\left(1+(\gamma \delta)^{2 k+1}\right) \\
& =2\left(1-\varphi_{2}^{2(2 k+1)}\right) .
\end{aligned}
$$

Since $\varphi_{0}=-1, L\left(f^{2 k}\right)=0$, and hence the theorem is proved.

A connected solvable Lie group $S$ is called of type (NR) (for 'no roots') if the eigenvalues of $\operatorname{Ad}(x): \mathfrak{S} \rightarrow \mathfrak{S}$ are always either equal to 1 or else they are not roots of unity. Solvable Lie groups of type (NR) were considered first in Keppelmann and McCord [3]. Since our solvmanifold $\Gamma \backslash \mathrm{Sol}_{1}^{4}$ is of type (NR), we have the following.

Theorem 5.2 Let $f: \Gamma \backslash \operatorname{Sol}_{1}^{4} \rightarrow \Gamma \backslash \operatorname{Sol}_{1}^{4}$ be a continuous map with linearization $\Phi_{*}=$ $\operatorname{diag}\left\{\varphi_{0}, \varphi_{1}, \varphi_{2}\right\}$. Then we have 
(1) Iff is of type (I), then

$$
\mathrm{NP}_{n}(f)=\mathrm{N} \Phi_{n}(f)=0 .
$$

(2) Suppose $f$ is of type (II). Then we have:

(a) If $n$ is odd and $\varphi_{2}= \pm 1$, then $\mathrm{NP}_{n}(f)=\mathrm{N} \Phi_{n}(f)=0$.

(b) If $n$ is odd and $\varphi_{2} \neq \pm 1$, then

$$
\mathrm{NP}_{n}(f)=2 \sum_{m \mid n} \mu(n / m)\left|1-\varphi_{2}^{2 m}\right|, \quad \mathrm{N} \Phi_{n}(f)=2\left|1-\varphi_{2}^{2 n}\right| .
$$

(c) If $n=2^{r} n_{0}$ with $n_{0}$ odd, then

$$
\mathrm{NP}_{n}(f)=0, \quad \mathrm{~N} \Phi_{n}(f)= \begin{cases}0 & \text { when } \varphi_{2}= \pm 1 \\ 2\left|1-\varphi_{2}^{2 n_{0}}\right| & \text { when } \varphi_{2} \neq \pm 1\end{cases}
$$

(3) Iff is of type (III), then

$$
\mathrm{NP}_{n}(f)=\sum_{m \mid n} \mu(n / m)\left|1-\varphi_{0}^{2 m}\right|, \quad \mathrm{N} \Phi_{n}(f)=\left|1-\varphi_{0}^{2 n}\right|
$$

Proof In case (1), the Nielsen number $N\left(f^{m}\right)=0$ for all positive integers $m$. The map $f$ has no essential periodic orbit classes of any period. It follows that $\mathrm{NP}_{n}(f)=\mathrm{N} \Phi_{n}(f)=0$.

Since $\Gamma \backslash \mathrm{Sol}_{1}^{4}$ is a solvmanifold of type (NR), by [2], Theorem 1.2, we have $\mathrm{N} \Phi_{m}(f)=$ $N\left(f^{m}\right)$ and $\mathrm{NP}_{m}(f)=\sum_{q \mid m} \mu(q) N\left(f^{\frac{m}{q}}\right)$ for all $m \mid n$ provided $N\left(f^{n}\right) \neq 0$. This proves our case (2)(b) and case (3) because of the following reason: When $f$ is of type (III), $N\left(f^{n}\right) \neq 0$; when $f$ is of type (II), $N\left(f^{n}\right)=\left(1-(-1)^{n}\right)\left|1-\varphi_{2}^{2 n}\right|$, and $N\left(f^{n}\right) \neq 0$ if and only if $n$ is odd and $\varphi_{2} \neq \pm 1$. In case (2) when $n$ is odd and $\varphi_{2}= \pm 1$, i.e., in case (2)(a), we note that $N\left(f^{m}\right)=0$ for all positive integers $m$. Hence $\mathrm{NP}_{n}(f)=\mathrm{N} \Phi_{n}(f)=0$.

Consider the case (2) with $n$ even. Since $N\left(f^{n}\right)=0$, it follows that $\mathrm{NP}_{n}(f)=0$. Let $n=2^{r} n_{0}$ for odd $n_{0}$. By Proposition 5.1, $f^{q}$ has no essential fixed point class for every even factor $q$ of $n$. Thus, the set of essential fixed point classes of $f^{q}$ with $q \mid n$ is the same as that of $f^{q}$ with $q \mid n_{0}$. Thus, $\mathrm{N}_{n}(f)=\mathrm{N} \Phi_{n_{0}}(f)$, which is just $N\left(f^{n_{0}}\right)=2\left|1-\varphi_{2}^{2 n_{0}}\right|$ if $\varphi_{2} \neq \pm 1$ and 0 if $\varphi_{2}= \pm 1$.

\section{Homotopy minimal periods}

In this section, we shall present the homotopy minimal periods for all maps on fourdimensional solvmanifolds $\Gamma \backslash \mathrm{Sol}_{1}^{4}$, which is of type (NR). Our main tool is

$$
\operatorname{HPer}(f)=\left\{n \mid N\left(f^{n}\right) \neq 0, N\left(f^{n}\right) \neq N\left(f^{\frac{n}{q}}\right) \text { for all prime } q \mid n\right\}
$$

This formula can be obtained immediately from the following theorem.

Theorem 6.1 ([21], Theorem 6.1) Let $f: M \rightarrow M$ be a self-map on a compact PL-manifold $M$ of dimension $\geq 3$. Then $f$ is homotopic to a map $g$ with $P_{n}(g)=\emptyset$ if and only if $\mathrm{NP}_{n}(f)=0$. 
Table 1 Homotopy minimal periods

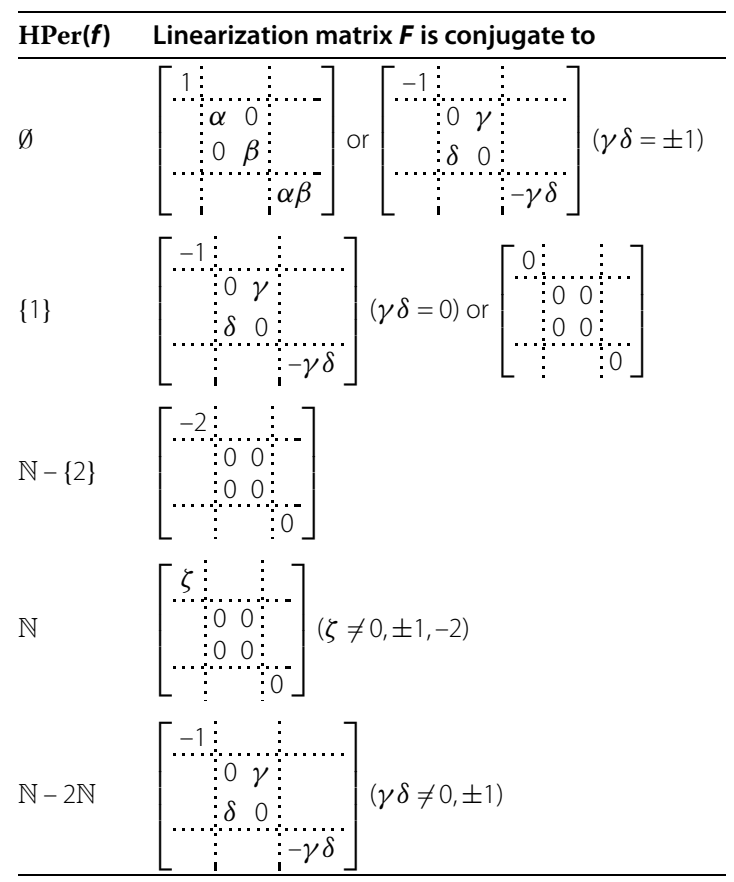

Proposition 6.2 ([9], Proposition 3.2) Let $f: M \rightarrow M$ be a self-map of a compact solvmanifold $M$ of type (NR). Then $\mathrm{NP}_{n}(f)=0$ if and only if either $N\left(f^{n}\right)=0$ or $N\left(f^{n}\right)=N\left(f^{n / q}\right)$ for some prime factor $q \mid n$.

Now the following is one of our main results.

Theorem 6.3 Let $f: \Gamma \backslash \operatorname{Sol}_{1}^{4} \rightarrow \Gamma \backslash \operatorname{Sol}_{1}^{4}$ be a continuous map with linearization $\Phi_{*}=$ $\operatorname{diag}\left\{\varphi_{0}, \varphi_{1}, \varphi_{2}\right\}$. Then we have

$$
\operatorname{HPer}(f)= \begin{cases}\{1\} & \text { when } \varphi_{0}=0 \\ \emptyset & \text { when } \varphi_{0}=1 \\ \varnothing & \text { when } \varphi_{0}=-1 \text { and } \varphi_{2}= \pm 1 \\ \{1\} & \text { when } \varphi_{0}=-1 \text { and } \varphi_{2}=0 \\ \mathbb{N}-2 \mathbb{N} & \text { when } \varphi_{0}=-1 \text { and } \varphi_{2} \neq 0, \pm 1 \\ \mathbb{N}-\{2\} & \text { when } \varphi_{0}=-2 \\ \mathbb{N} & \text { otherwise, i.e., } \varphi_{0} \neq 0, \pm 1,-2\end{cases}
$$

Proof (1) If $\varphi_{0}=0$, then $f$ is of type (III) and $N\left(f^{n}\right)=1$ for all $n$ by Proposition 5.1 . Hence $\operatorname{HPer}(f)=\{1\}$.

(2) If $\varphi_{0}=1$, then $f$ is of type (I) and so $N\left(f^{n}\right)=0$ for all $n$. This implies that HPer $(f)=\emptyset$.

(3) If $\varphi_{0}=-1$, then $f$ is of type (II). By Proposition $5.1, N\left(f^{n}\right)=0$ for all even $n$. It follows that $\operatorname{HPer}(f)$ does not contain any even number, i.e., $\operatorname{HPer}(f) \subset \mathbb{N}-2 \mathbb{N}$. Let us consider its subcases:

(3-1) If $\varphi_{2}=\operatorname{det} \varphi_{1}= \pm 1$, by Proposition $5.1, N\left(f^{n}\right)=0$ for all $n$. Thus HPer $(f)=\emptyset$. 
(3-2) If $\varphi_{2}=\operatorname{det} \varphi_{1}=0$, by Proposition 5.1, $N\left(f^{n}\right)=2$ for all odd $n$. Since $N(f) \neq 0$, we have $1 \in \operatorname{HPer}(f)$. By $(\mathrm{H})$, we have $n \notin \operatorname{HPer}(f)$ for all odd $n$ with $n>1$. Thus $\operatorname{HPer}(f)=\{1\}$.

(3-3) If $\varphi_{2}=\operatorname{det} \varphi_{1} \neq 0, \pm 1$, by Proposition 5.1, $N\left(f^{n}\right)^{\prime}$ 's are all distinct for all odd $n$. Hence $\operatorname{HPer}(f)=\mathbb{N}-2 \mathbb{N}$.

(4) If $\varphi_{0}=-2$, by Proposition 5.1, we have $N\left(f^{n}\right)=\left|1-(-2)^{n}\right|$. Especially, $N(f)=N\left(f^{2}\right)=$ 3. By $(\mathrm{H}), 1 \in \operatorname{HPer}(f)$ but $2 \notin \operatorname{HPer}(f)$. Since $N\left(f^{n+1}\right)>N\left(f^{n}\right)$ for all $n>2$, (H) induces that $\operatorname{HPer}(f)=\mathbb{N}-\{2\}$.

(5) Consider finally the case where $\varphi_{0} \neq-2,-1,0,1$. By Proposition 5.1 again, we still have $N\left(f^{n}\right)=\left|1-\varphi_{0}^{n}\right|$. In this case, we have that $N\left(f^{n+1}\right)>N\left(f^{n}\right)$ for all $n$. $(\mathrm{H})$ induces that $\operatorname{HPer}(f)=\mathbb{N}$.

We tabulate this result according to linearizations in Table 1. In fact, for each subset $S \subset \mathbb{N}$ appearing as $\operatorname{HPer}(f)$ and each form of linearization $\Phi_{*}$ listed above, there exists a self-map $f: \Gamma \backslash \operatorname{Sol}_{1}^{4} \rightarrow \Gamma \backslash \operatorname{Sol}_{1}^{4}$ such that $\operatorname{HPer}(f)=S$.

\section{Competing interests}

The authors declare that they have no competing interests.

\section{Authors' contributions}

All authors contributed equally and significantly in writing this paper. All authors read and approved the final manuscript.

\section{Acknowledgements}

The second-named author was supported by Basic Science Researcher Program through the National Research Foundation of Korea(NRF) funded by the Ministry of Education (No. 2013R1A1A2058693). The authors would like to thank the referees for making careful corrections to a few expressions and computations in the original version of the article.

Received: 4 June 2015 Accepted: 15 September 2015 Published online: 26 September 2015

\section{References}

1. Jiang, B: Lectures on Nielsen Fixed Point Theory. Contemp. Math., vol. 14. Am. Math. Soc., Providence (1983)

2. Heath, PR, Keppelmann, E: Fibre techniques in Nielsen periodic point theory on nil and solvmanifolds I. Topol. Appl. 76, 217-247 (1997)

3. Keppelmann, E, McCord, C: The Anosov theorem for exponential solvmanifolds. Pac. J. Math. 170, 143-159 (1995)

4. Kim, HJ, Lee, JB, Yoo, WS: Computation of the Nielsen type numbers for maps on the Klein bottle. J. Korean Math. Soc. 45, 1483-1503 (2008)

5. Ha, KY, Lee, JB: The Nielsen type numbers for maps on a 3-dimensional flat Riemannian manifold. Topol. Methods Nonlinear Anal. 45, 327-362 (2015)

6. Alsedà, L, Baldwin, S, Llibre, J, Swanson, R, Szlenk, W: Minimal sets of periods for torus maps via Nielsen numbers. Pac. J. Math. 169, 1-32 (1995)

7. Jezierski, J, Marzantowicz, W: Homotopy minimal periods for maps of three-dimensional nilmanifolds. Pac. J. Math. 209, 85-101 (2003)

8. Lee, JB, Zhao, X: Nielsen type numbers and homotopy minimal periods for all continuous maps on the 3-nilmanifolds. Sci. China Ser. A 51, 351-360 (2008)

9. Jezierski, J, Kȩdra, J, Marzantowicz, W: Homotopy minimal periods for NR-solvmanifolds maps. Topol. Appl. 144, 29-49 (2004)

10. Lee, JB, Zhao, $X$ : Nielsen type numbers and homotopy minimal periods for maps on the 3-solvmanifolds. Algebr. Geom. Topol. 8, 563-580 (2008)

11. Filipkiewicz, RO: Four dimensional geometries. Ph.D. thesis, University of Warwick (1984)

12. Wall, CTC: Geometric structures on compact complex analytic surfaces. Topology 25, 119-153 (1986)

13. Lee, KB, Thuong, S: Infra-solvmanifolds of Soli1-geometry. J. Korean Math. Soc. (to appear)

14. Dekimpe, K, Igodt, P, Kim, S, Lee, KB: Affine structures for closed 3-dimensional manifolds with Nil-geometry. Q. J. Math. 46, 141-167 (1995)

15. Ha, KY, Lee, JB: Crystallographic groups of Sol. Math. Nachr. 286, 1614-1667 (2013)

16. Wilking, B: Rigidity of group actions on solvable Lie groups. Math. Ann. 317, 195-237 (2000)

17. Lee, JB, Lee, KB: Averaging formula for Nielsen numbers of maps on infra-solvmanifolds of type (R). Nagoya Math. J. 196, 117-134 (2009)

18. Ha, KY, Lee, JB: Corrigendum to 'Averaging formula for Nielsen numbers of maps on infra-solvmanifolds of type (R)'. Nagoya Math. J. (to appear)

19. Ha, KY, Lee, JB, Penninckx, P: Anosov theorem for coincidences on special solvmanifolds of type (R). Proc. Am. Math. Soc. 139, 2239-2248 (2011)

20. McCord, CK: Lefschetz and Nielsen coincidence numbers on nilmanifolds and solvmanifolds II. Topol. Appl. 75, 81-92 (1997)

21. Jezierski, J: Wecken theorem for fixed and periodic points. In: Handbook of Topological Fixed Point Theory, pp. 555-615. Springer, Dordrecht (2005) 